\title{
IMPACT OF THE ENVIRONMENT AND SOCIETY ON ROBOTICS INNOVATIONS
}

\author{
Atef A. ATA ${ }^{1} \square$ (iD), N. M. Fonseca Ferreira $2 \square$ \\ ${ }^{1}$ Department of Engineering Mathematics And Physics, Faculty of Engineering, Alexandria University, \\ Alexandria (21544), Egypt \\ ${ }^{2}$ Engineering Coimbra Polytechnic - Isec, Coimbra, Portugal
}

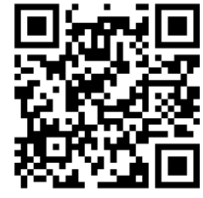

\section{ABSTRACT}

Received 01 August 2021

Accepted 15 August 2021

Published 11 November 2021

\section{CorrespondingAuthor}

Atef A.ATA, atefa@alexu.edu.eg

DOI 10.29121/IJOEST.v5.i6.2021.216

Funding: This research received no specific grant from any funding agency in the public, commercial, or not-for-profit sectors.

Copyright: (C) 2021 The Author(s). This is an open access article distributed under the terms of the Creative Commons Attribution License, which permits unrestricted use, distribution, and reproduction in any medium, provided the original author and source are credited.

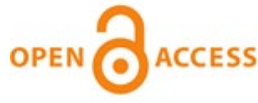

Over engineering history, environmental and social needs inspire the development and innovation of many beautiful applications. For example, when industrial robots entered our industrial life, significant advances, especially in the automotive industry, where industrial robots cover about $90 \%$ of the car industry activities, started to change our lives. Elderly people around the globe are increasing rapidly, and this requires us to focus on their needs as their children leave them alone in our busy daily life. Mechatronics and robotics can offer many possibilities to help older people by providing intelligent solutions for their daily needs and entertaining them during their lonely extended stay at their homes. Many companies provide intelligent mobile robots in different platforms (wheeled or walking) to help the elderly depend on themselves in receiving their food and medicine at prescheduled times. Other companies are interested in providing smart wheelchairs to help elderly people navigate indoor and outdoor freely and conveniently without any external help. Meanwhile, some companies are providing entertainment robots in different shapes to talk, play and communicate with elderly people in an excellent way. This paper aims to explore the mechatronics and robotics capabilities to assist elderly people and make their lives easier, comfortable, and self-governing without any external help. This paper will also highlight the effect of the environment and the social needs in inspiring innovations.

This paper will also discuss the idea and motivation behind two of Al-Jazari's robotic applications (Although the term robot was not coined at that time). These two devices are the washing hands and the peacock fountain. Although he used to apply mechanical structure and fluid for controlling the motion of the two devices, they were terrific and resemble some of the robotic applications nowadays.

Keywords: Environment, Robotics, Impact, Social, Innovations, Engineering.

\section{INTRODUCTION}

According to the statistics from the United Nations report shown in Figure 1, Figure 2 the number of elderly people over the age of 65 will increase dramatically in the coming four decades all over the world. This number is increasing in the advanced countries compared to the third-world countries since they have highly efficient healthcare programs to take care of elderly people. The situation is less problematic in third world countries, where many chronic diseases are still there, and many people die because of a lack of efficient health care programs and efficient medications and drugs. An example of this increase is shown in Figure 1 for India and China, the most populated countries in the world. 
According to the same source, we can conclude the following two figures for Europe and the United States as follows:

Youth vs. old age

The increasing proportions of aged persons show steady declines in the proportion of young persons. People 60 years old and over will be 32\% of Europe's total population by 2050 .

USA Figures:

- By 1900 there will be 3 million people on and above 65 years old.

- By 2010 more than 40 million people were on and above 65 years old.

- Around 2050, more than 88.5 million people on and over 65 years old and older.

It can also be observed from Figure 3 that the number of youth is decreasing while the number of elderly people is increasing rapidly.

The mechatronics and robotics fields have been contributing continuously to the benefits of elderly people for almost 40 years. This contribution can be classified into three main categories as follows:

Growth of the Population Aged 65 and Older in India and China:

2010-2050

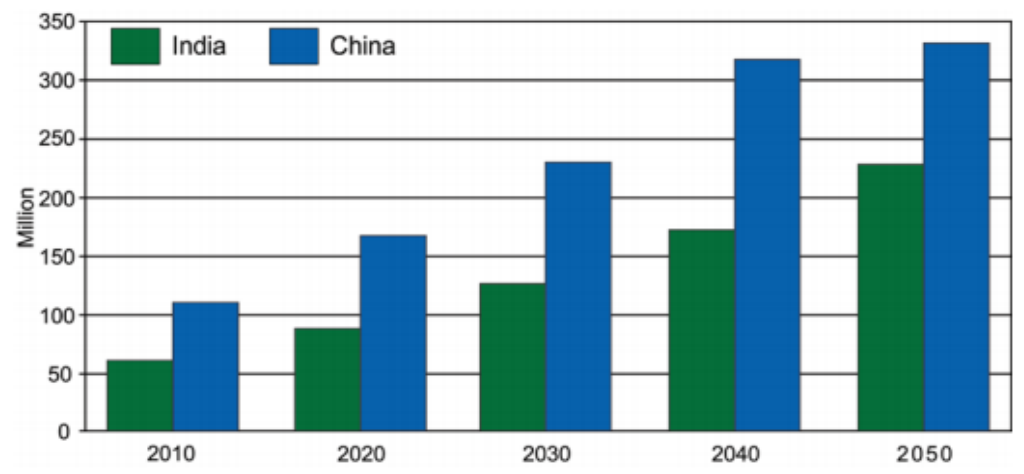

Source: United Nations. World Population Prospects: The 2010 Revision.

Available at: http://esa.un.org/unpd/wpp.

Figure 1 Population growth of people on and above 65 years old and older in India and China 20102050 [United Nations (2019)]

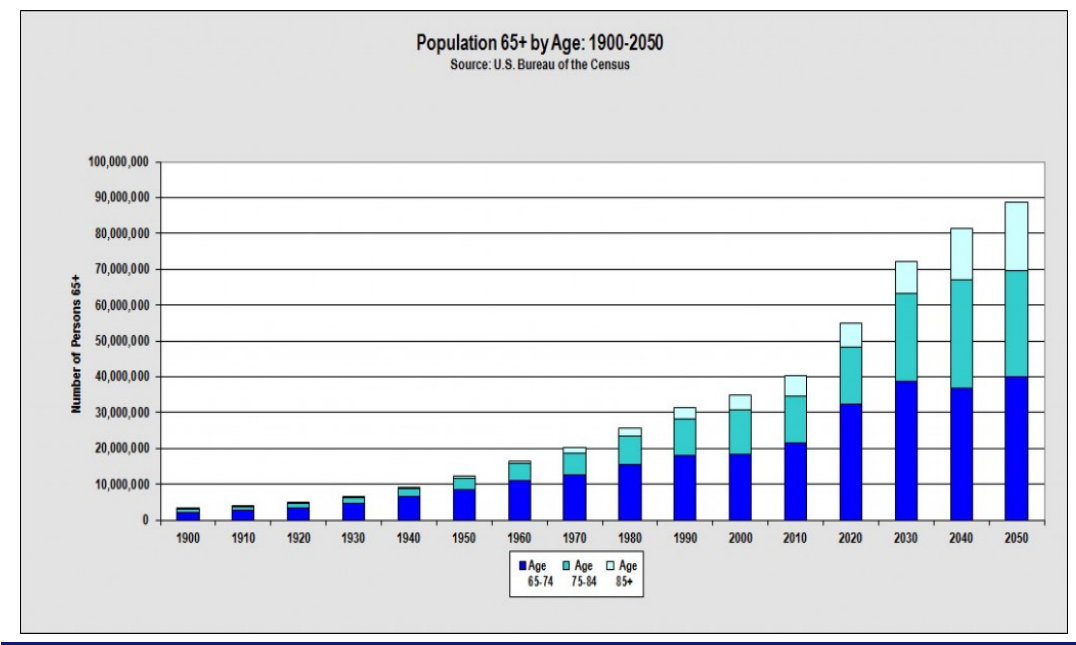

Figure 2 Population of people 65 years older and over is projected to reach 72 million in 2030 (Administration of Aging: Department of Health and Human Services) [Computers and Robots (2010)] 

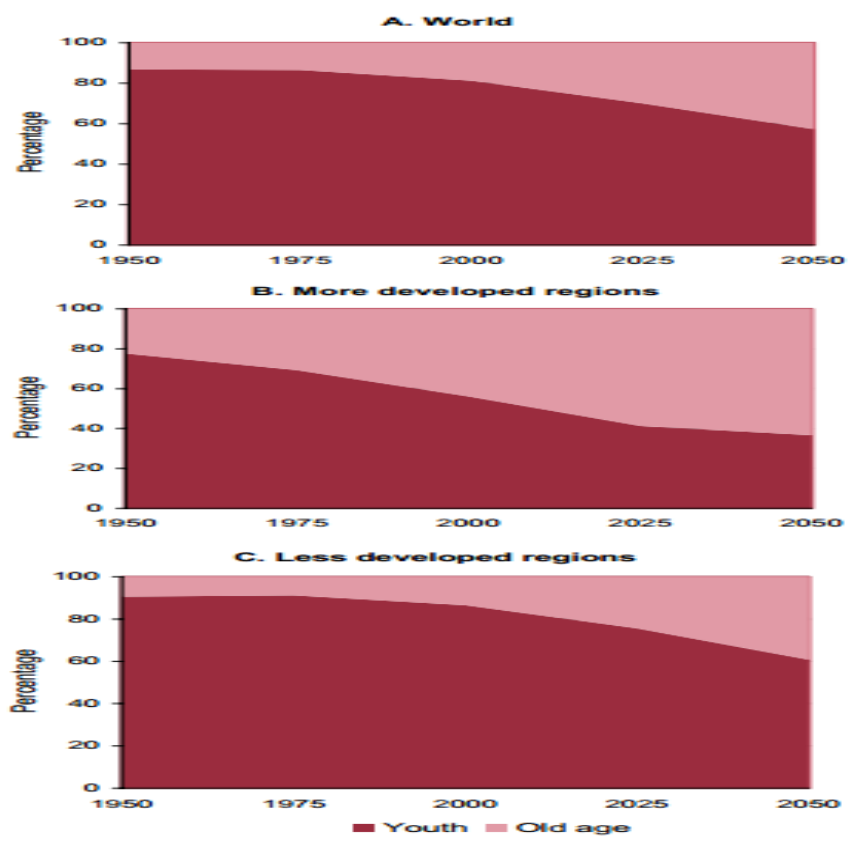

Figure 3 Total dependency ratio composition: world and development regions, 1950-2050 Source: Population Division, DESA, [United Nations (2019)]

1) Health care robots include autonomous nursing robots for medical assistance and bright stick for a blind for safe navigation.

2) Innovative wheelchairs: For the smooth mobility of the elderly and sick people, highly developed due to the contemporary advancement of personal computers and microcontrollers.

3) Entertainment robots and devices: This category has been developed recently to include talking and communicating robots such as AIBO from SONY, walking robots such as ASIMO from Honda, and the French robot NAO.

4) Robots for social and religious activities.

For the developed countries, the existence of mechatronic and robotic applications for elderly people is well known, and great numbers of research funds have been devoted to developing these applications. On the other hand, the third world countries can benefit from these developments by buying the final product if people can afford their high prices. For example, intelligent wheelchairs and disabled intelligent vehicles are available for rich people to buy and benefit from advanced technology.

This paper explores the existing and developed applications to assist elderly people in indoor and outdoor daily activities. It will also explore the existing problems regarding man-machine interaction and the possible solutions and future trends. The organization of this paper is as follows: Section (2) focuses on health care robots for elderly people, while section (3) investigates the advancements of intelligent wheelchairs for the benefit of elderly people. Then, section (4) highlights the entertainment examples that could help the elderly people enjoying their stay at their homes. Finally, section (5) investigates the design of robots to meet social and 
religious requirements, followed by the concluding remarks in section (6) and references.

\section{ELDERLY CARE ROBOTS}

The nursing robot applications for elderly people have been extensively increased for the last three decades. One of the famous nursing robots is the Helpmate, which is fabricated to be excellent in fetching and carrying tasks to imitate human behavior during motion inside the crowded hospital corridors. It can deliver trays, sterile supplies, medications, medical records, reports, samples, and late meals. Moreover, a helpmate has a friendly user interface by the help screen, turn signals, physical stops, and warning lights, enabling accurate and fast interaction with the controller [Krishnamurthy and Evans (1992)].

The fundamental motivation behind the design and development of Helpmate is saving a considerable amount of time and effort by nurses and supporting staff. The Institution Research Corporation aims to develop a wheeled mobile robot platform that can navigate crowded corridors, use lifts, and distribute materials to a reprogrammable position through the hospitals. Replacing nurses with robots in performing these time-consuming tasks, the staff can perform critical duties that robots cannot do in hospital rooms and operation theatres [Evans et al. (1989) ].

Although Japan is famous for designing and developing robots for elderly people, the Japanese government does not appreciate what robotic companies can produce. The main reason is that most of the companies do not consider the relevant people's remarks since they consider some of the products were too large and others are too expensive to buy.

The researchers at Toyohashi university of technology in Japan developed a nursing robot called "Terapio." Terapio is the robotic medical assistant that can help gather patient data and vital signs and relieve nurses from some of their work. Using Terapio enables nurses to have more devoted time to patients [http://www.extremetech.com/extreme/207130-terapio-autonomous-medicalrobot-can-assist-nurses ].

Some patients are unable to move or turn in their beds due to problems in their nervous systems. The latest generation of RIBA, the Robobear medical assistant robot, was introduced in Japan to overcome this problem. It is mainly designed to softly lift patients into or out of their beds and change their positions from sitting to standing and vice versa. It can also move the patient from the wheelchair and place him in his bed gently [http://www.robobear.org/].

Another interesting mechatronic innovation is the intelligent stick, which is developed to guide a visually impaired person to navigate freely and avoid obstacles during his walking around. The device consists of a microcontroller and a low-cost ultrasonic range finder to estimate the distance to any obstacle in the course of motion and give a feedback signal (Beeps or vibration) if it is near. These devices have become more popular now, and many electronic travel aids have been designed and developed to help blind people move independently and safely. The majority of these devices apply GPS technology to locate the position of the blind person. These systems can be used outdoor to trace the correct position of blind people (Normally in the form of coordinates) in case of emergencies. Different technologies have been utilized in recent years, such as RFID, Radio-Frequency Identification, Ultrasonic, GSM, and Laser [Gurubaran et al. (2014)].

\section{SMART WHEELCHAIRS}


A person with locomotive disabilities is known as handicapped, and he needs a wheelchair to help him move around indoors or outdoors. There are two types of wheelchairs: Manual and Smart. In the manual wheelchair, the person uses his hand and muscles to move it. Because many handicapped cannot use their hands due to weakness in their upper limbs, the concept of intelligent wheelchairs has been introduced in the last two decades. Smart wheelchairs offer many possibilities for the disabled to navigate, and some of them have other options such as feeding and submitting water and medications. The intelligent wheelchair can be controlled by either Bio-signal or non-Bio-signal techniques [Yadav and Sheoran (2016)].

The smart wheelchairs for elderly people can be classified into three main categories as follows:

3.1 Electric Wheelchair: These wheelchairs are designed for people who do not propel manual chairs. They can offer comfortable tilt, leg elevation, and seat elevation [Prasad et al. (2013)].

3.2 Standing wheelchairs: They are designed mainly for people with spinal cord injury. The most crucial characteristic is increasing cognition, well-being, and independence [Arva et al. (2009)].

3.3 Stair climbing wheelchair: This type of wheelchair has supporting devices that elevate the wheelchair and another set of supporting systems. The main disadvantage of this type is that it requires a wide stairway which is very expensive. Nevertheless, if the facilities have elevators, this would help solve the problems of support devices [Nirmal (2014)].

Due to the recent development in Human-Computer Interaction (HCI) and Human-Machine Interaction (HMI), many control techniques are available for intelligent wheelchairs. Among these techniques are Head Orientation Tracking Technique [Pajkanović and Dokić (2013)], Eye Tracking Technique [Chern-Sheng et al. (2006)], Tongue Controlled [Ruíz-Serranoa et al. (2013)], Touch Screen Controlled [Posugade et al. (2012) ], and Voice Actuated [Aruna et al. (2014)]. For more information regarding the control strategies of the innovative chairs, the reader is referred to the comprehensive review paper [Yadav and Sheoran (2016)].

Although smart wheelchairs provide high mobility for the disabled, the present systems lack observing the surrounding environment and the health conditions of most of the patients. Another drawback is the cost versus accuracy, which is very important for most patients. In addition, some of the advanced wheelchairs are under the research evaluation phase, and they need some time to be available in the market.

\section{ENTERTAINMENT ROBOTS}

SONY designed and developed AIBO as an entertainment robot pronounced eye-bow, meaning companion in the Japanese language. The main objective of AIBO is to behave like a robotic pet capable of interacting with its human owner in the same way normal pets usually do. According to SONY, AIBO is an autonomous robot that can learn, mature, and behave by itself in response to external excitations. The human interaction causes AIBO to express its emotions, needs, ability to learn, and maturity. It can also express many human-like feelings such as happiness, sadness, surprise, fear, anger, and dislike. Similar to live pets, as long as AIBO interacts with humans, it will learn faster.

AIBO has been equipped with many sensors such as a Touch sensor for nonverbal communication, a stereo microphone to hear, a color video cam for vision, and a distance detector to avoid collision with nearby objects. For AIBO to communicate verbally with its owner, it has been equipped with voice recognition 
components that can be programmed to recognize its name and about 50 verbal commands. It is a bi-lingual to understand both Japanese and English languages, and SONY plans to add more languages such as German and French to increase the robot's recognition internationally. Another exciting feature of AIBO is its ability to interact with humans by emitting musical tones and changing the shape and color of its eyes. AIBO also can be programmed to play games to entertain elderly people [http://searchcio.techtarget.com/definition/AIBO ].

Softbank, a French company, introduced NAO as an entertainment robot. Although AIBO is fabricated from metal, it can respond to spoken commands. As a result, it was an efficient rehabilitation tool in treating severely demented patients [Tamura et al. (2004)].

Although it is designed to accompany a person in his daily life with its small size and beautiful round edges, each NAO has its personality. Equipped with tremendous programming tools, it has various applications in both education and research in many international institutions [https://www.ald.softbankrobotics.com/en/cooleobots].

Softbank introduced two more humanoid robots for entertainment Pepper and Romeo. Pepper is a humanoid platform personal and emotional robot that can read emotions. It welcomed the visitors at many sales stores and was available to the general public in countries such as Japan. On the other hand, Romeo is a platform for exploration to develop innovative technologies and solutions for disabled people. It is the fruit of the scientific collaboration of many European laboratories and institutions mainly to assist people in their daily activities.

The Honda Motor Company wanted to create a walking robot that would help people around the house. As a result, Advanced Step in Innovative Mobility (ASIMO) was developed as the most advanced humanoid robot. ASIMO has 57 degrees of freedom for easy maneuvering and mobility. According to its developer, ASIMO can walk independently without any human assistance in a stable way. ASIMO was designed to understand spoken commands by humans and preprogrammed gestures. It can also recognize voices and faces and can be interfaced with IC communication cards. ASIMO has arms and hands imitating humans, and it can perform some normal human behavior such as turning on the lights, opening doors, carrying objects, and pushing carts.

ASIMO can also help elderly people, mainly if someone is confined to a wheelchair or bed. It was designed to be $1.3 \mathrm{~m}$ tall to look eye to eye with a seated person on a chair. ASIMO's dimensions enable it to carry out its designated work without being too big. Its external skeleton looks friendly and non-threatening to work well for the purpose its manufacturer had in mind before developing it [http://science.howstuffworks.com/asimo.htm].

\section{ROBOTS FOR RELIGIOUS ACTIVITIES}

The Czech writer Carl Capek first coined the word robot in his play Rossum's Universal Robot in 1920 to denote a fictional humanoid. The word Robot in Czeck's language refers to a forced worker. Isaak Asimov, a Russian Physician, took the same approach and created the three laws of robotics in a series of science fiction novels by 1936. Three eminent scientists, William Grey Walter, George Devol, and Joseph Engelberger, are considered the founder of robotics today. George Devol invented Unimate, the first digital and programmable robot, in 1954, and it was sold to General Motors to be used in material handling of hot metals from the die casting machine [https://historiafactory.wordpress.com/2016/06/19/al-jazari-11361206-the-founder-of- biotics/ ]. 
Arabs and Muslims contributed to robotics a long time ago, as illustrated by Mark E. Rosheim (1944). Badi Az-Zaman Ismail Al-Jazari was a Muslim scientist born in 1136 and lived in Diyar-Bakir (South east of Turkey now) enhanced robotic engineering during the $12^{\text {th }}$ Century, although the word robot was not known at that time. Al-Jazari was also a polymath, mathematician, mechanical engineer, inventor, and artisan. Al-Jazari, like his father before him, served as chief engineer at the Artuklu Palace of a Turkish dynasty of Artukids who ruled in Eastern Anatolia and Jazira in the $12^{\text {th }}$ and $13^{\text {th }}$ centuries [Rosheim (1994)].

Al-Jazari (1136-1206) commissioned and compiled "The Science of Ingenious Mechanisms," which consists of two parts: Compilation or variation of existing designs and his inventions. Al-Jazari may have constructed many of the mechanisms mentioned in his text in 1206, including educational and entertaining, and presented them to the Sultan. In addition, he described modern labour-saving devices and unusual clocks. Some of them can be considered as his designs [http://www.muslimheritage.com/article/al-jazari-mechanical-genius.].

Inspired by the environment and society, Al-Jazari started his robotic innovation. Although Al-Jazari did not call his inventions robots, they can resemble robot structures from our own real life. He depended mainly on mechanical movement, and he used fluid for control which replaces the temporary microcontroller devices nowadays. His innovations were inspired by the religious activities and culture where Muslims eat by their hands, not spoons, and they need to wash their hands after eating. Another necessity is ablution which every Muslim has to do five times a day before each prayer. In this paper, two compelling examples of how the environment and the social needs affect the design and function of the innovation are illustrated. Among these mechanisms, two designs are illustrated here [Uzun et al. (2008)].

The first design is a device to aid in washing hands. Figure 4 shows a kneeling female with a large ewer in her hand by a basin filled with water. There is also a bird on the top of the device. Once the bird whistles, water pours into a basin below. A duck then drinks the used water and releases it through its tail into contained under the platform. When the user pulls the lever, water drains. This design anticipates the modern flush toilet we are using today.

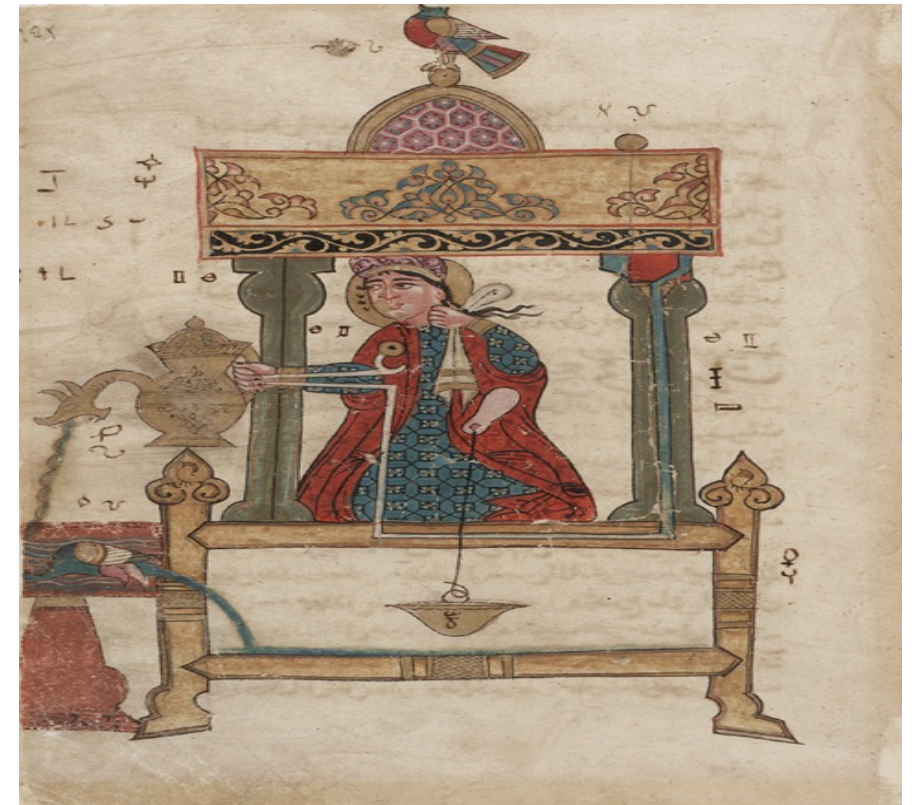

Figure 4 Washing hands device. (C) The Smithsonian Institution, Washington [Rosheim (1994), http://www.muslimheritage.com/article/al-jazari-mechanical-genius.]. 
The second device is the peacock fountain, where Al-Jazari also made a robotic man for the Sultan. This robot, modeled on a 12-year old boy, provided water, a towel, and a comb for the Sultan during his ablutions, as shown in Figure 5. Pulling a plug on the Peacock's tail releases water out of the peak; as the dirty water from the basin fills the hollow base, a float rises and actuates a linkage, making a servant figure appear from behind a door under the peacock and offer soap.

When more water is used, a second float at a higher level trips and causes the appearance of a second servant figure- with a towel. Opening the base valve causes both figures to return to their room, and the doors automatically close as the water level drops.

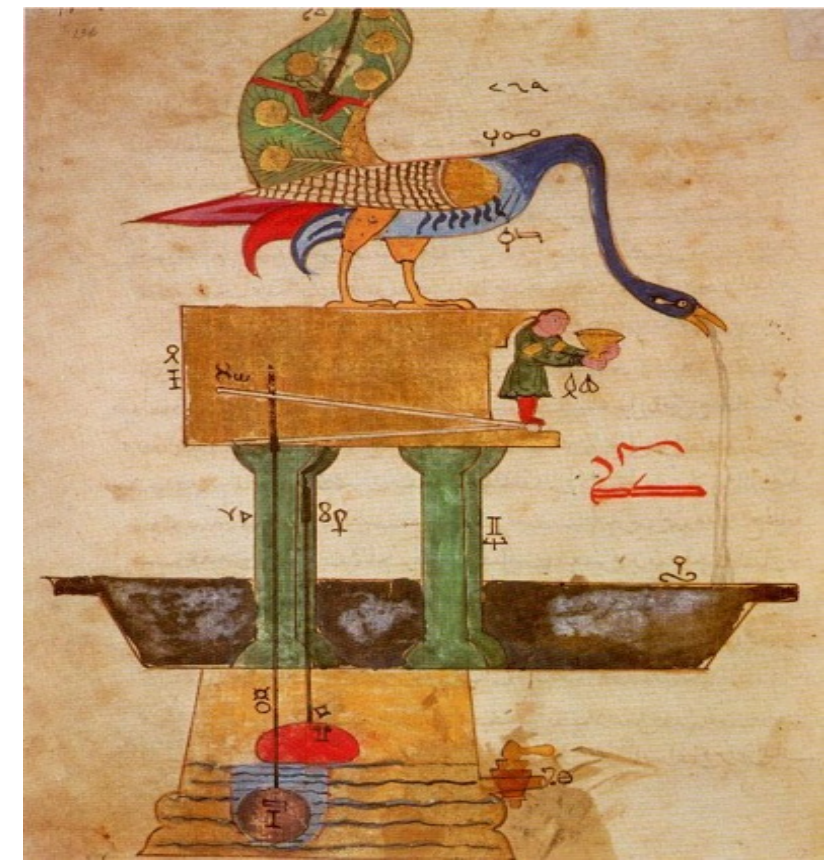

Figure 5 Wash-basin in the form of a peacock described by Al-Jazari in Kitab fi Ma'rifat al-Hiyal alHandisayya [Rosheim (1994), http://www.muslimheritage.com/article/al-jazarimechanical-genius.].

\section{CONCLUSIONS}

The contribution of mechatronics and robotics to serve elderly people to make their lives easier and more convenient has been presented. Also, some problems regarding how elderly people react to the new technology and some possible solutions are presented. The interaction between the elderly people and the machine (Man-Machine Interaction) should be designed carefully and safely. People are still afraid to deal with the machines and always request a user-friendly Graphic User Interface (GUI). Also, when it comes to surgical robots like MINERVA or Da Vinci, people have some concerns about giving their consent when they know that a robot will do the operation with a robot-assisted surgeon. Awareness about the accuracy of robots and their safety requirements should be spread among elderly people since they accepted the smart wheelchairs as they saw by their own eyes their benefits for mobility and other tasks. By good advertisements, similar devices can have the same trust and success. 
cameras to supervise the device and also the surrounding, to supervise the device while in use to stop it in case of an emergency. Also, adding a deadlock switch to most autonomous devices in the range of elderly people is advisable to deal with unscheduled situations.

\section{REFERENCES}

Aruna C, Dhivya Parameswari A, Malini M, Gopu G. (2014) Voice recognition and touch screen control based wheelchair for paraplegic persons. IEEE, 1-5. Retrieved from https://doi.org/10.1109/ICGCCEE.2014.6922215

Arva J, Paleg G, Lange M, Lieberman J, Schmeler M, Dicianno B et al. (2009) RESNA Position on the application of wheelchair standing devices. Taylor \& Francis; 21: 161-168. Retrieved from https://doi.org/10.1080/10400430903175622

Chern-Sheng L, Chien-Waho, Wen-Chen, Chuang-Chien C, Mav-Shiun Y. (2006) Powered wheelchair controlled by the eye-tracking system. Optica Applicata; XXXVI 2-3: 401-412.

Computers and Robots (2010): Decision-Makers in an Automated World, Retrieved from https://cs.stanford.edu/people/eroberts/cs201/projects/201011/Computers Making Decision/ welcome/index.html.

Evans J, Krishnamurthy B, Pong W, King S, Weiman C., Bogardus G, et al. (1989) Help Mate: a service robot for health care. Industrial Robot: An International Journal 1989; 16, 2:87 - 89. Retrieved from https://doi.org/10.1108/eb005047

Gurubaran, Kasilingam G, Ramalingam M. (2014) A survey of voice aided electronic stick for visually impaired people, International Journal of Innovative Research in Advanced Engineering (IJIRAE); 1, 8:342-346.

Krishnamurthy B, Evans J. (1992) HelpMate: A robotic courier for hospital use", Proceedings of the IEEE International Conference on Systems, Man, and Cybernetics, 18-20 October 1992, Chicago, 1630-1634.

Nirmal TM. (2014) Wheelchair for physically and mentally disabled persons. International Journal of Electrical and Electronics Research; 2, 2: 112-118.

Pajkanović A, Dokić B. (2013) Wheelchair control by head motion. Serbian Journal of Electrical Engineering; 10:1 135-151. Retrieved from https://doi.org/10.2298/SJEE1301135P

Posugade VG, Shedge KK, Tikhe CS. (2012) Touch-screen based wheelchair system", International Journal of Engineering Research and Applications. IJERA; 2: 2, 1245-1248.

Prasad A, Shah S, Ruparelia P, Sawant A. (2013) Powered wheelchairs. International Journal of Scientific \& Technology Research; 2, 11: 162-165.

Rosheim M E. (1994) Robot Evolution: The Development of Anthrobotics. John Wiley and Sons, New York.

Ruíz-Serranoa A, Posada-Gómeza R, Sibajaa MA, Rodrígueza GA, Gonzalez-Sancheza BE, Sandoval-Gonzaleza 00. (2013) Development of a dual control system applied to a smart wheelchair, using magnetic and speech control. Iberoamerican Conference on Electronics Engineering and Computer Science ELSEVIER; 7, $158 \quad$ - 165. Retrieved from https://doi.org/10.1016/j.protcy.2013.04.020

Tamura T., S. Yonemitsu, A. Itoh, D. Oikawa, A. Kawakami, Y. Higashi, T. Fujimoto, and K. Nakajima (2004)," Is an Entertainment Robot Useful in the Care of 
Elderly People With Severe Dementia, Journal of Gerontology; 59A: 1, 83-85. Retrieved from https://doi.org/10.1093/gerona/59.1.M83

United Nations (2019), World Population Prospects: The 2010 Revision, Retrieved from http://esa.un.org/unpd/wpp

Uzun A, Vatansever F. Ismail AL Jazzari (2008) Machines and New Technologies, Acta Mechanica et Automatica; 2: 3, 91-94.

Yadav S, Sheoran P. (2016) Smart wheelchairs: A literature review. International Journal of Innovative and Emerging Research in Engineering; 3, 2: 86-90.

http://science.howstuffworks.com/asimo.htm.

http://searchcio.techtarget.com/definition/AIBO

http://www.extremetech.com/extreme/207130-terapio-autonomous-medicalrobot-can-assist-nurses

http://www.muslimheritage.com/article/al-jazari-mechanical-genius.

http://www.robobear.org/.

https://historiafactory.wordpress.com/2016/06/19/al-jazari-1136-1206-thefounder-of- biotics/

https://www.ald.softbankrobotics.com/en/cool-eobots. 